\title{
Rotary Ventricular Assist Device Control With a Fiber Bragg Grating Pressure Sensor
}

\author{
Andrew F. Stephens ${ }^{\circledR}$, Andrew Busch, Member, IEEE, Robert F. Salamonsen, \\ Shaun D. Gregory, and Geoffrey D. Tansley
}

\begin{abstract}
Current ventricular assist devices (VADs) are rotary blood pumps used to treat end-stage heart failure. VADs are operated at a constant speed that is manually adjusted by a clinician based on the patient's cardiac demand during routine medical examinations. VADs operated at a constant speed have inadequate passive flow regulation due to the inherent mechanical pressure-flow characteristics of the pump; this can lead to harmful situations where the VAD is overpumping or underpumping. Typically, patients on long-term VAD support are discharged to an outpatient setting where the VAD speed can remain the same for weeks or months at a time, impacting patient safety and quality of life. Previously, physiological controllers for VADs have been proposed, which automatically adjust VAD speed to meet patient cardiac demand. Clinical implementation of physiological control is currently hindered by the lack of clinically available, implantable, and continuous hemodynamic sensors. This study describes the physiological control of a VAD using a fiber Bragg grating (FBG) sensor previously developed for measuring VAD inlet pressure. The FBG sensor was used as a feedback to a Starling-like physiological controller, and the control quality was compared against the same controller with feedback from a nonimplantable industrial pressure sensor (Omega sensor). Experiments were conducted in a bench-top cardiovascular simulator under various simulated patient scenarios. The average steady-state difference in VAD flow across all experiments was $0.1 \mathrm{~L} / \mathrm{min}$ with a maximum difference of $-0.4 \mathrm{~L} / \mathrm{min}$. Similarly, the average steady-state difference in left ventricular end-diastolic pressure was $0.02 \mathrm{mmHg}$ with a maximum difference of $\mathbf{- 0 . 2} \mathbf{~ m m H g}$. The clinically insignificant
\end{abstract}

Manuscript received July 25, 2019; revised January 19, 2020; accepted April 13, 2020. Manuscript received in final form April 17, 2020. This work was supported in part by The Prince Charles Hospital Foundation under Grant NI2015-220 and Grant TM2017-04, in part by the National Health and Medical Research Council under Grant APP1079421, in part by Griffith University, in part by Monash University, and in part by the Baker Heart and Diabetes Institute. The work of Shaun D. Gregory was supported by the National Heart Foundation, Australia, under Grant 102062. Recommended by Associate Editor K. Barton. (Corresponding author: Andrew F. Stephens.)

Andrew F. Stephens and Shaun D. Gregory were with the School of Engineering and Built Environment, Griffith University, Mount Gravatt, QLD 4122, Australia. They are now with the Department of Mechanical and Aerospace Engineering, Monash University, Clayton, VIC 3800, Australia, and also with the Cardiorespiratory Engineering and Technology Laboratory, Baker Heart and Diabetes Institute, Melbourne, VIC 3004, Australia (e-mail: research.andrew.stephens@gmail.com; shaun.gregory@monash.edu).

Andrew Busch and Geoffrey D. Tansley are with the School of Engineering and Built Environment, Griffith University, Brisbane, QLD 4122, Australia (e-mail: a.busch@griffith.edu.au; g.tansley@griffith.edu.au).

Robert F. Salamonsen is with the Department of Epidemiology, Monash University, Clayton, VIC 3800, Australia, and also with the Intensive Care Unit, Alfred Hospital, Melbourne, VIC 3004, Australia (e-mail: robert.salamonsen@monash.edu).

Color versions of one or more of the figures in this article are available online at http://ieeexplore.ieee.org.

Digital Object Identifier 10.1109/TCST.2020.2989692 differences found between the two feedback methods indicate that the FBG pressure sensor is viable for the physiological control of VADs.

Index Terms-Fiber Bragg grating (FBG), implantable pressure sensor, physiological control, ventricular assist device (VAD).

\section{INTRODUCTION}

$\mathbf{H}$ EART transplantation is the current gold-standard treatment for end-stage heart failure. However, the demand for donor's hearts far exceeds the supply. Ventricular assist devices (VADs) can be used to treat end-stage heart failure while awaiting transplant, or as a "destination" therapy. State-of-the-art rotary VADs are implantable blood pumps that draw blood from the failing ventricle and pump it back into the body to provide short- or long-term cardiac support. Advances in rotary VAD technology and medical management over the last three decades has improved the one-year survival rate of VAD supported patients to rival that of heart transplant recipients [1], [2]. This has led to a shift in research priority away from patient survival and into the patient quality of life, which has not seen any significant improvement over the last decade [3]-[5].

During VAD treatment, a complicated medical management regime is employed to ensure patient comfort and safety, with adjustment of VAD speed being a critical management parameter. Currently, VADs are run at a constant speed that is set manually by a skilled clinician based on the blood flow requirements (cardiac demand) of the patient during routine medical examinations. It has been shown that VADs operated at a constant speed have an inadequate response to changes in preload (the amount of blood that has filled the ventricle before contraction) compared with the native heart that is highly sensitive to changes in preload [6]. The preload insensitivity of VADs is a consequence of the flow-pressure-head characteristics of the pump and results in an inability of the VAD to passively adapt its outflow in response to changes in cardiac demand. This, in turn, can lead to situations where the VAD is underpumping, leading to pulmonary congestion where blood can pool in the lungs, or overpumping, which can lead to ventricular suction, where the VAD draws too much blood from the ventricle and the pump sucks down on the ventricular chamber. Similarly, the lack of preload responsiveness of the VAD does not allow it to automatically respond to changes in patient states, as occurring throughout a typical day. This can 
affect the patient's capacity to exercise, sleep, change posture, or complete any number of other autonomous daily activities, leading to a reduction in patient quality of life.

In the clinical setting, the patient is closely monitored, and the VAD speed is adjusted, as necessary, to meet patient cardiac demand. However, currently, 53\% of VAD patients are supported for more than one year [1], and subsequently, many patients are discharged to an outpatient setting, which results in a significant decrease in the frequency of VAD speed management $(\approx 1-6$ weeks at the author's institution depending on the patient). Outpatients also more likely have varied metabolic demand compared with their hospitalized counterparts, leaving them more susceptible to pump-related adverse events, such as suction or congestion [7]. Therefore, it is desirable for VADs to have some mechanism, which can actively vary VAD speed, and therefore flow, to meet changes in patient cardiac demand and avoid harmful overpumping and underpumping.

Various physiological control systems have been previously proposed, which aim to automatically modify VAD speed based on any number of hemodynamic or electromechanical variables, such as blood pressure, blood flow, heart rate, and VAD power consumption. One such physiological controller is Starling-like control that mimics the healthy heart's response to changes in preload, as determined by the measured pressure in the left ventricle before contraction (left ventricular enddiastolic pressure or $\left.P_{\mathrm{LVED}}\right)$. Starling-like control has been shown to improve the preload sensitivity of the VAD, avoid simulated ventricular suction and congestion, and reduce ventricular loading during exercise [8]-[10]; however, one of the main limitations of Starling-like control, which requires pressure and flow data for decision making, is the lack of clinically available, implantable hemodynamic sensors.

Currently, there is one implantable flow sensor for VADs, which is clinically available - the FlowAccurate for the ReliantHeart aVAD (ReliantHeart Inc., NY, USA). The FlowAccurate continuously records VAD outflow and stores it to the VAD controller for upload via a cellular network. While clinical results have varied using the FlowAccurate [11], [12], it represents an important step toward the realization of physiological control. Meanwhile, only one implantable blood pressure transducer has been approved for clinical use. The CardioMEMS (St. Jude Medical, MN, USA) is an implantable pulmonary artery pressure monitor. Unfortunately, the CardioMEMS is passively actuated, via external excitation, and does not provide continuous readings, making it unsuitable for physiological control applications. Therefore, an implantable, continuous pressure transducer that is compatible with VADs is highly desirable.

Several experimental pressure transducers have been designed for VADs [13]-[16], but none of these have seen clinical translation. Following many successful years of deployment in structural, civil, and aerospace applications, the recent adoption of fiber optic sensing technologies in the medical sector has given rise to exciting new opportunities for hemodynamic sensing [17]. Previously, the authors published a study that characterized a novel fiber optic pressure sensor that was designed specifically for VADs [18]. The previously investigated fiber optic sensor consists of a single-mode silica fiber embedded into a flexible silicone cylinder that employs the fiber Bragg gratings (FBGs) as sensing elements.

In this study, the control capabilities of the FBG sensor are explored by combining the FBG pressure sensor with a Starling-like physiological controller for VADs. The FBG sensor was used as the feedback to the Starling-like controller (SLC) for decision making and evaluated on a physical bench-top cardiovascular simulator. The quality of control provided by the SLC using FBG pressure sensor feedback was quantitatively compared against an SLC using pressure feedback provided by a commercially available nonimplantable pressure sensor. Differences in steady-state error and transient controller operating behavior were used to demonstrate any clinically significant differences between the two feedback methods under a variety of perturbations to a simulated patient.

\section{Materials AND Methods}

\section{A. Sensing Principle and Sensor Design}

The FBG pressure sensor was described in detail in a previous work [18]. Briefly, the sensor is comprised of a flexible silicone cylinder that has an optical fiber embedded into it. Two FBGs are written into the core of the optical fiber and are used as sensing elements. An FBG is a periodic diffraction grating that can reflect specific wavelengths of light $\lambda_{B}$ with respect to the periodicity of the Bragg grating [19]. Equation (1) demonstrates the effect of strain and temperature on $\lambda_{B}$ where strain causes changes in the periodicity of the Bragg grating segments $(\Lambda)$, and the changes in temperature will cause variations in $n_{\text {eff }}$, the relative refractive index of the fiber

$$
\lambda_{B}=2 \cdot n_{\mathrm{eff}} \cdot \Lambda \text {. }
$$

Equation (1) can be, otherwise, represented as a linear change in $\lambda_{B}$ with respect to temperature, strain, and straininduced temperature due to the thermal expansion of the material that the fiber is embedded into. These variations can then be converted into a desired measurand by creatively embedding the FBG into different materials under different configurations. In this case, the FBG fiber is embedded into a compliant silicone cylinder, which can be situated in series with the VAD inlet or outlet and is in contact with the blood passing through the VAD. Because the cylinder is compliant, changes in blood pressure cause the cylinder to balloon inward or outward, and the ballooning motion is sensed as a strain by the FBG, which, once calibrated, can be converted to measure the blood pressure. Small physiological variations in body temperature also facilitate the need to adjust the FBG pressure sensor readings according to changes in body temperature. This is achieved by using the second FBG sensing element, which is isolated from strain by a mechanical splice protector but is still able to detect changes in temperature. A temperature compensation algorithm is then generated based on the thermal and strain coefficients of the FBG sensor [20]. The conversion from $\lambda_{B}$ to pressure for the temperature and strain sensing grating is given as

$$
P=a \lambda_{T}+b \lambda_{\epsilon}+c
$$




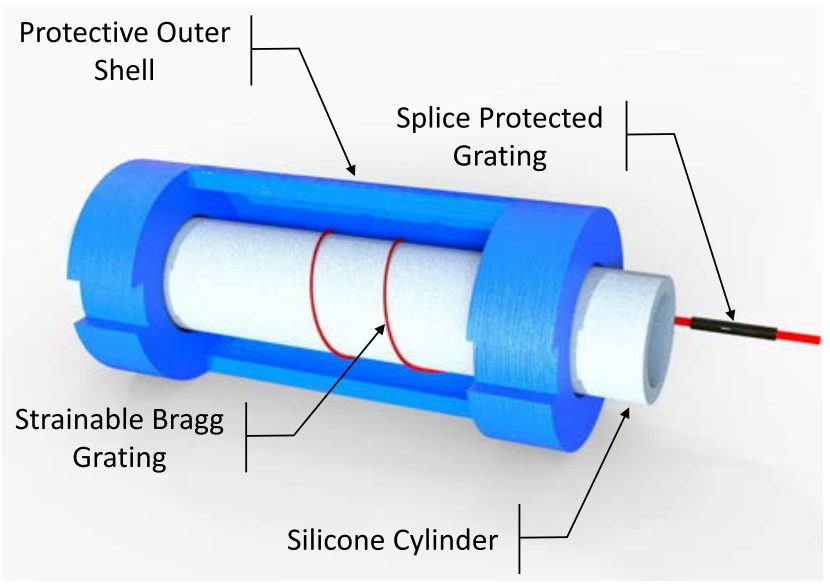

Fig. 1. Cross-sectional render of an FBG pressure sensor for VADs.

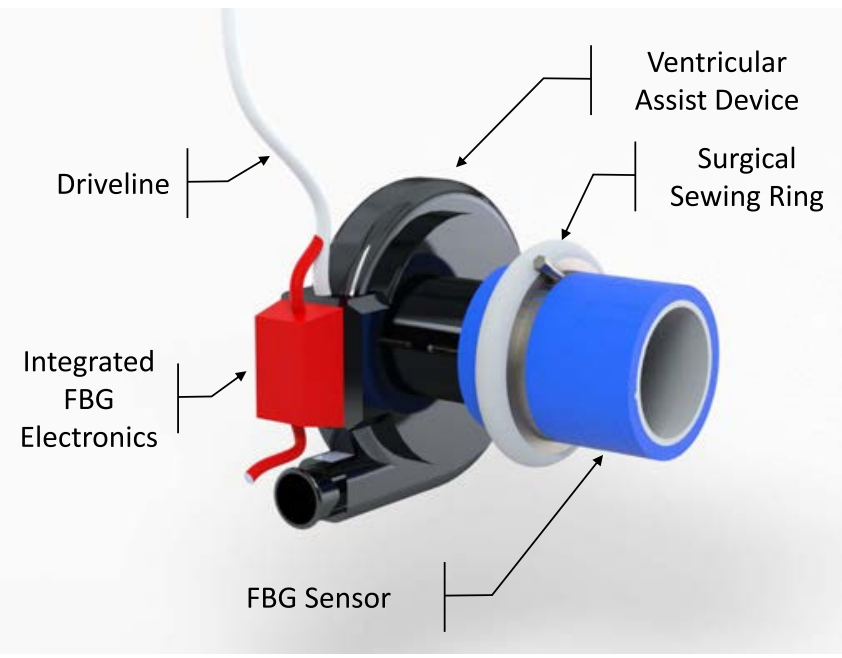

Fig. 2. Concept render of FBG pressure sensor and demodulation electronics integrated with the VAD.

where $\lambda_{T}$ is the Bragg wavelength of the temperature sensing element, $\lambda_{\epsilon}$ is the Bragg wavelength of the strain sensing element, and $a-c$ are calibration coefficients for a given sensor (in this study, $a=51.23, b=-46.108$, and $c=4852.7$ ). The FBG pressure sensor was previously found to have a range between -25 and $200 \mathrm{mmHg}$, a sensitivity of $-0.0046 \mathrm{~mm} / \mathrm{mmHg}$, an accuracy of $0.35 \mathrm{mmHg}$ at $37^{\circ} \mathrm{C}$, and a resolution of $0.018 \mathrm{mmHg}$ [18]. FBG interrogation, achieved by a spectrophotometer I-MON 256, Ibsen Photonics, Farum, Denmark, is experimentally found to be capable of accurately resolving changes in peak wavelength of $0.175 \mathrm{pm}$. A rendering of the FBG sensor is presented in Fig. 1, while a concept render of the sensor integrated with the VAD is presented in Fig. 2.

\section{B. Starling-Like Controller}

Starling-like control for VADs aims to automatically adjust VAD speed, and therefore flow, based on changes in patient cardiac demand. Starling-like control is described fully elsewhere [8], [21]. Briefly, Starling-like control mimics the native healthy flow regulating mechanism of the heart-the Starling mechanism. The Starling mechanism describes the function by which the heart varies cardiac output based on the amount of

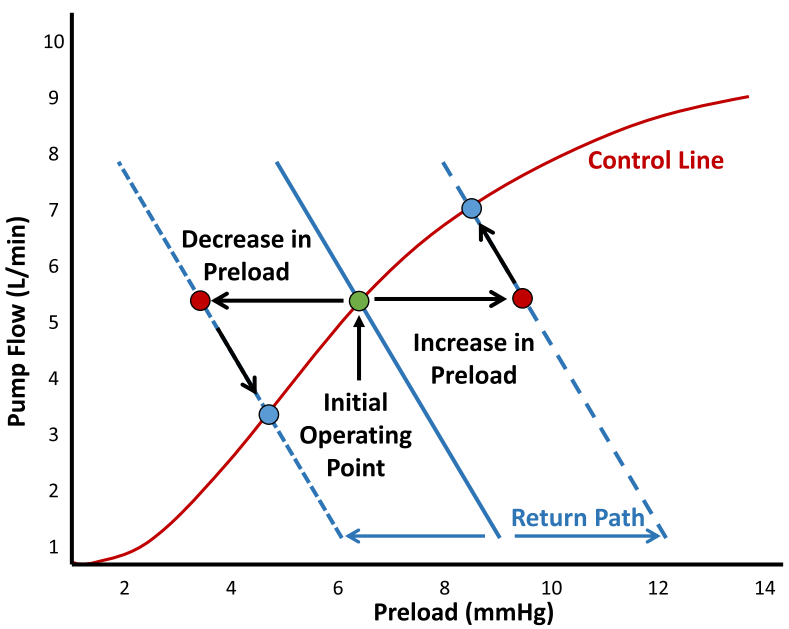

Fig. 3. Starling-like control emulates the native heart function through the establishment of a control line (representing the cardiac response function) and a return path (simulating venous return). Deviations in preload cause lateral shifts in the controller OP (green circle to red circles), and the OP is then stepped along the return path to the point of intersection with the control line, which is used as the new target VAD flow. The final OP is given by the blue circles.

blood returned to the ventricles prior to contraction [22]. Work by Guyton [23] and Sarnoff and Berglund [24] characterized the Starling mechanism through the establishment of cardiac output and venous return functions, which can be mathematically represented.

In a similar way, Starling-like VAD control emulates the cardiac output and venous return functions to predict the required VAD flow $\left(\overline{Q_{\mathrm{LVAD}}}\right)$ based on the preload in the ventricle as indicated by left ventricular end-diastolic pressure $\left(P_{\mathrm{LVED}}\right)$. In every heartbeat, the SLC determines the required VAD flow as the point of intersection between the control line (modeling the cardiac output function) and the return path (modeling the venous return function), as shown in Fig. 3. Deviations in preload cause the controller operating point (OP-combination of $P_{\mathrm{LVED}}$ and $\overline{Q_{\mathrm{LVAD}}}$ ) to be stepped along the return path until the OP intersects the control line. The value at the point of intersection is the predicted target flow ${\overline{Q_{\mathrm{LVAD}}}}^{*}$, and the proportional-integral control is then used to converge the target flow ${\overline{Q_{\mathrm{LVAD}}}}^{*}$ with the measured flow $\overline{Q_{\mathrm{LVAD}}}$. The control line is represented by (3) where $\overline{Q_{\mathrm{LVAD}}}$ * is the predicted VAD flow, $P_{\mathrm{LVED}}$ is the left ventricular enddiastolic pressure (preload), and SF is the scaling factor that modifies the overall power of the Starling-like function, as occurs in the healthy heart through inotropic variations

$$
{\overline{Q_{\mathrm{LVAD}}}}^{*}=\left(10.3+\left[\frac{-10.3}{1+\left(\frac{P_{\mathrm{LEED}}}{7}\right)^{2.3}}\right]\right) \cdot \mathrm{SF} .
$$

Modifying the scaling factor is achieved through the use of upper and lower $P_{\mathrm{LVED}}$ limits that tell the controller when the patient is in immediate danger (emergency adaption) outer black dashed lines (see Fig. 4). The controller also more frequently attempts to bring the patient back to within a clinician defined "optimized" level (sweet spot adaption) (inner blue dotted lines Fig. 4). In the case of emergency adaption, the controller modifies the scaling factor immediately once 


\section{Control Boxes for ASLC Feedback Experiments}

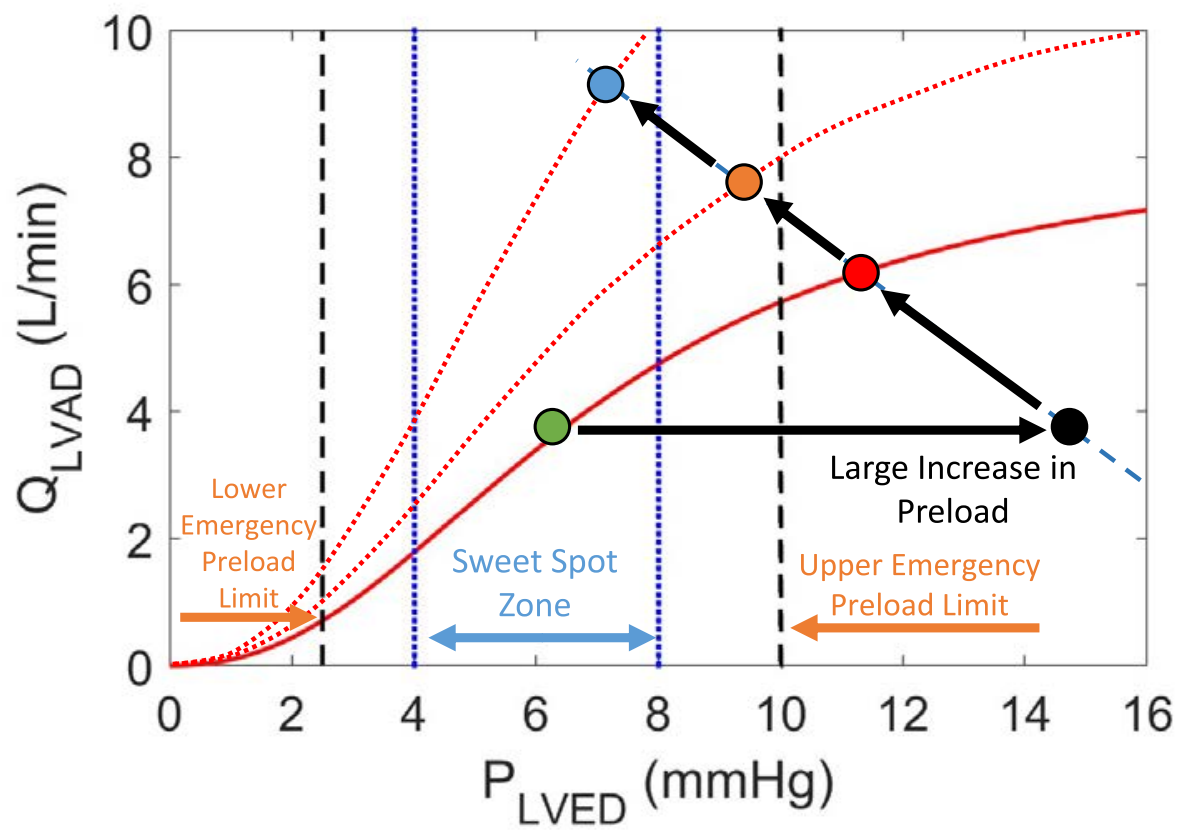

Fig. 4. SLC can adapt the control line (solid red line: original; dotted red lines: adapted control lines) to increase the power of the Starling-like mechanism. Emergency control line adaptions occur when the OP (green circle) travels outside of the emergency limits (dotted outer black lines). Emergency adaptions will bring the OP back to within acceptable limits (orange circle). Similarly, sweet spot adaptions will bring the OP to back within the sweet spot zone (blue circle).

the $P_{\text {LVED }}$ limit has been violated and the controller is in the steady state. Meanwhile, sweet spot adaption occurs if the OP is within the emergency limits but not within the sweet spot zone. Once the controller is in the steady state, sweet spot adaptions trigger every $20 \mathrm{~s}$ to bring the OP to back within the sweet spot limits. An example of an increase in the control line scaling factor due to both emergency and sweet spot adaption is given in Fig. 4.

For the experiments presented here, the scaling factor was initially set to 1 . A dead zone of $1 \%$ of the target $P_{\mathrm{LVED}}$ was used to indicate controller steady state. The return path gradient (modeling the venous return) is a simple linear relationship between $\overline{Q_{\mathrm{LVAD}}}$ and $P_{\mathrm{LVED}}$, which is used to predict to predict the steady-state ${\overline{Q_{\mathrm{LVAD}}}}^{*}$. The return path gradient was set to $-1.96 \mathrm{~L} / \mathrm{min} / \mathrm{mmHg}$ as determined by the internal cardiovascular simulator parameters [25]. The upper and lower emergency preload limits were set to 10 and $2.5 \mathrm{mmHg}$, respectively, for congestion and suction avoidance. The upper and lower sweet spot preload limits were set to 8 and $4 \mathrm{mmHg}$ on advice from a senior intensive care clinician from the author's institution.

\section{Mock Circulatory Loop}

All experiments were conducted on a physical bench-top cardiovascular simulator [mock circulatory loop (MCL)]. The MCL is a five-element Windkessel model including pulmonary and systemic circulations. The MCL contains four polyvinyl chloride (PVC) cylinders representing the aorta, pulmonary artery, systemic veins, and pulmonary veins. Four smaller PVC chambers that are pneumatically driven represent the left and right atria and ventricles. Mechanical check valves situated at the atria and ventricles ensure unidirectional flow throughout the circuit. Ventricular and atrial contraction is controlled through a series of electropneumatic regulators (ITV2030012BS5, SMC Corporation, Tokyo, Japan) and three/two-way solenoid valves (VT325-035DLS, SMC Corporation, Tokyo, Japan) to provide passively filled heart chambers. Heart rate and systolic fraction were maintained at 80 beats/min and $35 \%$, respectively, for each experiment, unless otherwise stated. Systemic and pulmonary vascular resistances (SVR and PVR) were controlled by electropneumatically actuated socket valves (VMP025.03X.71, AKO UK, Northamptonshire, U.K.). The working fluid was a water and glycerol mixture 60\%/40\% weight/weight, which mimicked the typical viscosity of blood (3.57 $\mathrm{mPa} \cdot \mathrm{s}$ at room temperature). A HeartWare HVAD (Medtronic, Dublin, Ireland) was connected to the MCL with the inflow cannulation site at the ventricle and the outflow cannulation site at the aorta. More information on the MCL is available in [26].

The MCL was set to simulate left heart failure with a functional "healthy" right ventricle (see Table I). The HVAD was used to restore the heart failure condition to simulated healthy levels by turning on the VAD and SLC and allowing the controller to reach a steady-state (see Table I). For the combined FBG pressure sensor and SLC (FBG-SLC), the FBG sensor was connected in series with the VAD inlet to measure $P_{\text {LVED }}$. The center of the FBG sensing element was positioned approximately $75 \mathrm{~mm}$ away from the ventricle and $75 \mathrm{~mm}$ from the VAD at the center of the inlet cannula (see Fig. 5). For comparison, an Omega pressure sensor (PX181B-015C5V, Omega Engineering, Norwalk, CT, USA) was also used to 


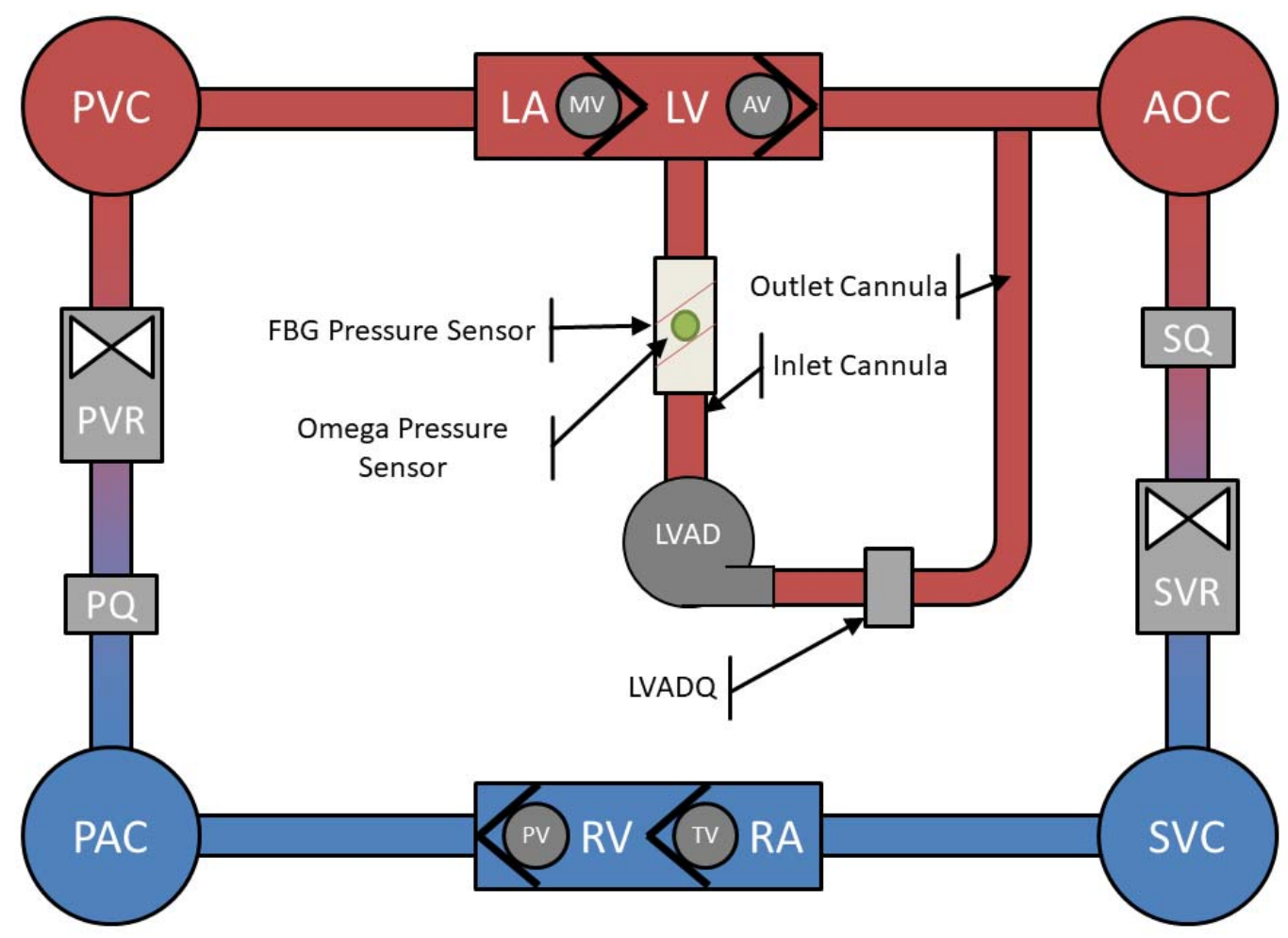

Fig. 5. Left VAD (LVAD) supported MCL, including FBG pressure sensor on the LVAD inlet cannula (white and red rectangle) and an Omega pressure sensor on the LVAD inlet cannula (green circle). LA and RA: left and right atria; LV and RV: left and right ventricles; MV, TV, AV, and PV: mitral, tricuspid, aortic, and pulmonary valves; AOC and PAC: aortic and pulmonary artery compliance chambers; SVC and PVC: systemic and pulmonary venous compliance chambers; SQ and PQ: systemic and pulmonary flow meters; SVR and PVR: systemic and pulmonary vascular resistance valves; and LVADQ: LVAD flowmeter.

TABLE I

Baseline Heart Failure (Bhf) and Restored Healthy Condition (RHC) Following LVAd Support. $\overline{P_{\text {Ao }}}$ : Mean Aortic Pressure; $\overline{P_{\mathrm{PA}}}$ : Mean Pulmonary Artery Pressure; CO: Mean Cardiac Output; $\overline{Q_{\mathrm{LV}}}$ : Mean LVAd Flow; $P_{\text {Lved }}$ : Left Ventricular EndDiastolic Pressure; $P_{\text {Rved }}$ : Right Ventricular End Diastolic Pressure; And $\omega_{\text {LVad }}:$ LVAD Speed

\begin{tabular}{cccccccc}
\hline Condition & $\begin{array}{c}\overline{P_{A O}} \\
(\mathrm{mmHg})\end{array}$ & $\begin{array}{c}\overline{P_{P A}} \\
(\mathrm{mmHg})\end{array}$ & $\begin{array}{c}\mathrm{CO} \\
(\mathrm{L} / \mathrm{min})\end{array}$ & $\begin{array}{c}\overline{Q_{L V A D}} \\
(\mathrm{~L} / \mathrm{min})\end{array}$ & $\begin{array}{c}P_{L V E D} \\
(\mathrm{mmHg})\end{array}$ & $\begin{array}{c}P_{R V E D} \\
(\mathrm{mmHg})\end{array}$ & $\begin{array}{c}\omega_{L V A D} \\
(\mathrm{rpm})\end{array}$ \\
\hline \hline BHF & 69.0 & 22.0 & 3.8 & - & 13.0 & 4.4 & - \\
RHC & 85.0 & 19.0 & 4.8 & 4.6 & 6.5 & 5.6 & 2600 \\
\hline
\end{tabular}

provide feedback to the SLC (Omega-SLC); this was achieved by replacing the FBG pressure sensor with the Omega pressure sensor on the VAD inlet, again approximately $75 \mathrm{~mm}$ away from the ventricle and VAD (see Fig. 5) to ensure the comparable pressure readings. The Omega sensor was used for comparison as it is the same sensor that provided feedback to the SLC in previous studies [8], [25]. Meanwhile, flow for the SLC was measured by a Transonic clamp on flowmeter (TS410-10PXL, Transonic Systems, NY, USA), which uses the same technology as clinically available implantable VAD flow sensors (FlowAccurate, ReliantHeart Inc., TX, USA). A separate Omega pressure sensor was used to monitor the MCL's left ventricular pressure.

All data were acquired into a dSPACE 1103 (dSPACE $\mathrm{GmbH}$, Paderborn, Germany) at a rate of $2 \mathrm{kHz}$ and downsampled to $200 \mathrm{~Hz}$ for postprocessing. The MCL's cardiovascular model was simulated within MATLAB's Simulink (MATLAB 2016b, The MathWorks Inc., MA, USA) and executed on the dSPACE 1103 at $2 \mathrm{kHz}$. The FBG and Omega pressure sensors were calibrated against a pressure gauge (DPG-1001B-15G,
Omega Engineering Inc., Norwalk, CT, USA), which was National Institute of Standards and Technology (NIST) traceable to within $\pm 0.5 \mathrm{mmHg}$. Signals from the FBG and Omega pressure sensors were fed into a LabJack U6 pro (LabJack Corporation, CO, USA) data acquisition system, and the appropriate gain was applied via a custom LabView model (virtual instrument) (LabView 2015 SP1, National Instruments, TX, USA). After amplification, the signals were then fed out of the LabJack via an analog output back into the dSPACE 1103, which was running the MCL and SLC models. Feeding both pressures out through the LabJack was done to allow the same level of digitization between the FBG and Omega sensors. The FBG pressure sensor was stable and only required minimal low-pass filtering $\left(\omega_{C}=0.05\right)$; for control stability, the Omega pressure sensor required more filtering $\left(\omega_{C}=0.125\right)$. The sampling time of the LabJack was $100 \mathrm{~Hz}$. $P_{\text {LVED }}$ was calculated in the dSPACE model by capturing the pressure reading immediately before systole was triggered in the MCL model. A block diagram showing the interface between the MCL and pressure sensors is presented in Fig. 6 . 


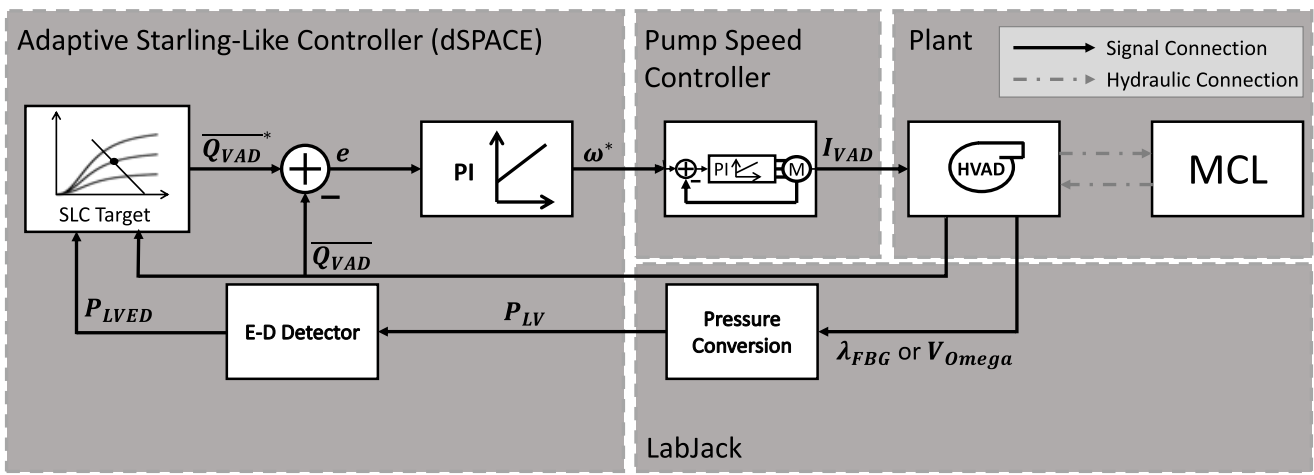

Fig. 6. Block diagram showing the interface between the SLC, VAD, and developed FBG sensor. $\overline{Q_{\mathrm{LVAD}}} *$ : target mean LVAD flow; $\overline{Q_{\mathrm{LVAD}}}$ : measured mean LVAD flow; $P_{\mathrm{LV}}$ : left ventricular pump inlet pressure; $P_{\mathrm{LVED}}$ : left ventricular end-diastolic pressure; $I_{\mathrm{VAD}}$ : VAD current; $\omega^{*}$ : target VAD speed; MCL: mock circulatory loop; $V_{\text {Omega }}$ : Omega pressure sensor voltage; $\lambda_{\mathrm{FBG}}$ : FBG wavelength; and E-D Detector: end-diastolic pressure detector.

\section{Experimental Protocol}

The FBG-SLC (FBG sensor as a feedback to the SLC) and Omega-SLC (Omega sensor as a feedback to the SLC) were tested under a variety of perturbations to the MCL simulating different patient states. The perturbations included changes in preload (through adjustments to the volume of the circulating fluid), changes in afterload (through modifications in SVR), and exercise (through modifications in preload, afterload, heart rate, and ventricular contractility) (see Table II). The pattern of experiments was to allow $10 \mathrm{~s}$ of baseline data, followed by a 30-s ramp to the test condition, allow $120 \mathrm{~s}$ for the controller and MCL to settle, followed by a 30-s ramp back to baseline and a further $60 \mathrm{~s}$ of steady state. The time values were chosen to ensure the MCL and SLC reached steady state between each transition as the SLC has a designed response time of between 30 and $60 \mathrm{~s}$, in the same order of magnitude as the healthy body's response to exercise [21], [27]. Each perturbation was conducted once using FBG-SLC and once using Omega-SLC due to the high repeatability of the MCL [26].

The transient and steady-state controller responses of the two feedback modalities were compared to identify any clinically significant difference between the two. In an attempt to quantify the difference between the feedback modes, a Gaussian shoelace formula [see (4)] was used to calculate the area inside the locus described by the OP migrations (OP path) for the onset of the experiment as well as the return to baseline. In (4), $n$ is the number of OP data points recorded, $P$ is the measured $P_{\mathrm{LVED}}$, and $Q$ is the measured VAD flow $\left(\overline{Q_{\mathrm{LVAD}}}\right)$. Equation (4) assumes that each OP data point acts as a discrete point in an irregular polygon and that the first and last OP data points in an experiment connect to make an enclosed surface. This method is useful for quantifying the difference between the OP paths of the two feedback modalities. However, the value obtained is a comparative approximation and should be taken as indicative only, as it is affected by noise

$A=\frac{1}{2} \cdot\left[\sum_{i=1}^{n-1} P \cdot Q_{i+1}+P_{n} \cdot Q_{1}-\sum_{i=1}^{n-1} P_{i+1} \cdot Q_{i}-P_{1} \cdot Q_{n}\right]$.
TABLE II

CHANGES IN MCL VARIABLES FOR EACH EXPERIMENT. SVR: SySTEMIC VASCUlar RESistance; PVR: PUlmonary VASCUlar RESISTANCE; HR: Heart Rate; and $\phi$ : Phase Shift. Note that 1 dyne $\cdot s$. $\mathrm{cm}^{-5}=1 \mathrm{MPa} \cdot \mathrm{s} \cdot \mathrm{m}^{-3}$

\begin{tabular}{ccc} 
Condition & Variable(s) & Value \\
\hline \hline Afterload Increase & SVR & $1300 \rightarrow 3000$ dyne $\cdot \mathrm{s} \cdot \mathrm{cm}^{-5}$ \\
Afterload Decrease & SVR & $1300 \rightarrow 600$ dyne $\cdot \mathrm{s} \cdot \mathrm{cm}^{-5}$ \\
Preload Increase & Circulating Fluid & $+0.5 \mathrm{~L}$ \\
Preload Decrease & Circulating Fluid & $-0.5 \mathrm{~L}$ \\
\hline & SVR & $1300 \rightarrow 700$ dyne $\cdot \mathrm{s} \cdot \mathrm{cm}^{-5}$ \\
& PVR & $160 \rightarrow 70$ dyne $\cdot \mathrm{s} \cdot \mathrm{cm}^{-5}$ \\
Exercise & HR & $80 \rightarrow 100 \mathrm{bpm}$ \\
& Volume & $+700 \mathrm{~mL}$ \\
& Contractility & $+10 \%$ \\
& Atrial Kick & $\phi 20 \%$ and 35\% systolic duty \\
\hline
\end{tabular}

\section{RESULTS}

Results for each of the experiments are presented in their appropriate sections. The results are tabulated in detail in Table III. For all postprocessing, $P_{\mathrm{LVED}}$ has been low-pass filtered using a cutoff frequency of $10 \mathrm{~Hz}\left(\omega_{C}=62.83\right)$ to make the OP paths easier to identify.

\section{A. Increase in Afterload}

During the increase in SVR, $\overline{Q_{\mathrm{LVAD}}}$ reduced due to the decrease in pump pressure head; in turn, the SLC increased the VAD speed. Both sensors provided a similarly shaped OP path (see Fig. 7) with the FBG-SLC delivering a larger OP path area $(1.09 \mathrm{~L} \cdot \mathrm{mmHg} / \mathrm{min})$ compared with the Omega-SLC OP path area $(0.91 \mathrm{~L} \cdot \mathrm{mmHg} / \mathrm{min})$ indicating a less "tight" control by the SLC. The steady-state $\overline{Q_{\mathrm{LVAD}}}$ was $4.2 \mathrm{~L} / \mathrm{min}$ for both control modalities. The steady-state error between the $P_{\text {LVED }}$ and the two sensors remained constant at $0.1 \mathrm{mmHg}$ (see Fig. 8).

\section{B. Decrease in Afterload}

During the decrease in SVR, $\overline{Q_{\mathrm{LVAD}}}$ increased due to the increase in pump pressure head; in response, the SLC reduced the VAD speed. Again, both sensors produced a similarly shaped OP path with the FBG-SLC providing a smaller OP 
TABLE III

Recorded Steady-State Hemodynamics for Each of the Conducted Experiments for Both of the Feedback Sensors. The Absolute Steady-State Difference Between a Pair of Experiments Is Given in Brackets For Each VARiable. FBG: FBG Sensor; OMega: Omega Sensor; $\overline{P_{\text {AO }}}$ : Mean Aortic Pressure; $P_{\text {lved }}$ : Left Ventricular End-Diastolic Pump Inlet Pressure; CO: Cardiac OutPut of The Left Ventricle; $\overline{Q_{\text {LVAD }}}$ : MeAn LVAD Flow; $\omega_{\text {LVAD }}$ : LVAD SPEed; AND OP AREA: AREA Within THE OP PATH

\begin{tabular}{ccccccc}
$\begin{array}{c}\text { Condition } \\
\text { (Feedback Sensor) }\end{array}$ & $\begin{array}{c}\overline{P_{A O}} \\
(\mathrm{mmHg})\end{array}$ & $\begin{array}{c}P_{L V E D} \\
(\mathrm{mmHg})\end{array}$ & $\begin{array}{c}\mathrm{CO} \\
(\mathrm{L} / \mathrm{min})\end{array}$ & $\begin{array}{c}\overline{Q_{L V A D}} \\
(\mathrm{~L} / \mathrm{min})\end{array}$ & $\begin{array}{c}\omega \cdot \mathrm{LVAD} \\
(\mathrm{rpm})\end{array}$ & $\begin{array}{c}\text { OP Area } \\
(L \cdot \mathrm{mmHg} / \mathrm{min})\end{array}$ \\
\hline \hline Baseline Heart Failure & 69.0 & 13.0 & 3.8 & - & - & - \\
Restored Healthy Condition & 85.0 & 6.5 & 4.8 & 4.6 & 2600 & - \\
Afterload Increase (FBG) & 170.9 & 6.1 & 4.3 & 4.2 & 3660 & 1.09 \\
Afterload Increase (Omega) & $169.9(1)$ & $6.0(0.1)$ & $4.3(0)$ & $4.2(0)$ & 3620 & $0.91(0.18)$ \\
Afterload Decrease (FBG) & 48.0 & 6.1 & 5.2 & 4.2 & 2080 & 0.86 \\
Afterload Decrease (Omega) & $47.9(0.1)$ & $6.0(0.1)$ & $5.2(0)$ & $4.1(0.1)$ & 2000 & $1.36(-0.5)$ \\
Preload Increase (FBG) & 98.6 & 7.2 & 5.4 & 5.2 & 2850 & 0.65 \\
Preload Increase (Omega) & $101.4(-2.8)$ & $7.4(-0.2)$ & $5.6(-0.2)$ & $5.4(-0.2)$ & 2930 & $0.86(-0.21)$ \\
Preload Decrease (FBG) & 67.1 & 5.6 & 3.7 & 3.7 & 2310 & 1.23 \\
Preload Decrease (Omega) & $67.7(-0.6)$ & $5.5(0.1)$ & $3.7(0)$ & $3.7(0)$ & 2325 & $1.68(-0.45)$ \\
Exercise (FBG) & 83.8 & 7.8 & 8.1 & 7.2 & 2980 & 9.4 \\
Exercise (Omega) & $85.1(-1.3)$ & $7.8(0)$ & $8.4(-0.3)$ & $7.6(-0.4)$ & 3000 & $10.3(-1.1)$ \\
\hline
\end{tabular}

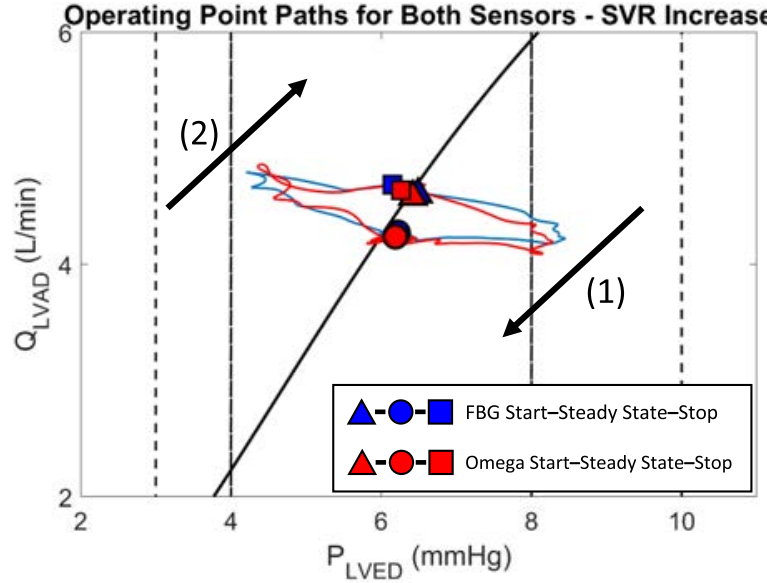

Fig. 7. SLC OP path using FBG transducer (FBG-blue) and Omega transducer (red) as a feedback variable during increases in SVR. The arrow labeled (1) indicates the OP path following the onset of the SVR increase, while the arrow labeled (2) indicates the OP path following the return of SVR to baseline. The markers indicate the start, steady state, and final positions of the OP. The solid black line is the Starling-like control line.

path area $(0.86 \mathrm{~L} \cdot \mathrm{mmHg} / \mathrm{min})$ compared with the OmegaSLC $(1.36 \mathrm{~L} \cdot \mathrm{mmHg} / \mathrm{min})$, indicating "tighter control" from the FBG sensor and less variation in preload. FBG-SLC steady-state $\overline{Q_{\mathrm{LVAD}}}$ was $3.4 \mathrm{~L} / \mathrm{min}$, only $0.1 \mathrm{~L} / \mathrm{min}$ higher than the steady-state provided by the Omega-SLC $\left(\overline{Q_{\mathrm{LVAD}}}\right.$ of $3.3 \mathrm{~L} / \mathrm{min}$ ). The steady-state difference in $P_{\mathrm{LVED}}$ between the Omega-SLC and FBG-SLC was maintained at $0.1 \mathrm{mmHg}$.

\section{Increase in Preload}

During the increase in preload, the SLC increased VAD speed and $\overline{Q_{\mathrm{LVAD}}}$ under feedback from both sensors. The OP path was again similar for both sensors; this time, the FBGSLC produced a smaller OP path area $(0.65 \mathrm{~L} \cdot \mathrm{mmHg} / \mathrm{min})$ than its Omega-SLC counterpart $(0.86 \mathrm{~L} \cdot \mathrm{mmHg} / \mathrm{min})$, again indicating "tighter" control and less variation in preload. The steady-state $\overline{Q_{\mathrm{LVAD}}}$ for the FBG-SLC was $5.2 \mathrm{~L} / \mathrm{min}$, while the Omega-SLC provided $5.4 \mathrm{~L} / \mathrm{min}$ steady-state flow. The steady state $P_{\text {LVED }}$ for the FBG-SLC and Omega-SLC feedback was 7.2 and $7.4 \mathrm{mmHg}$, respectively.

\section{End-diastolic Pressure for Both Sensors}
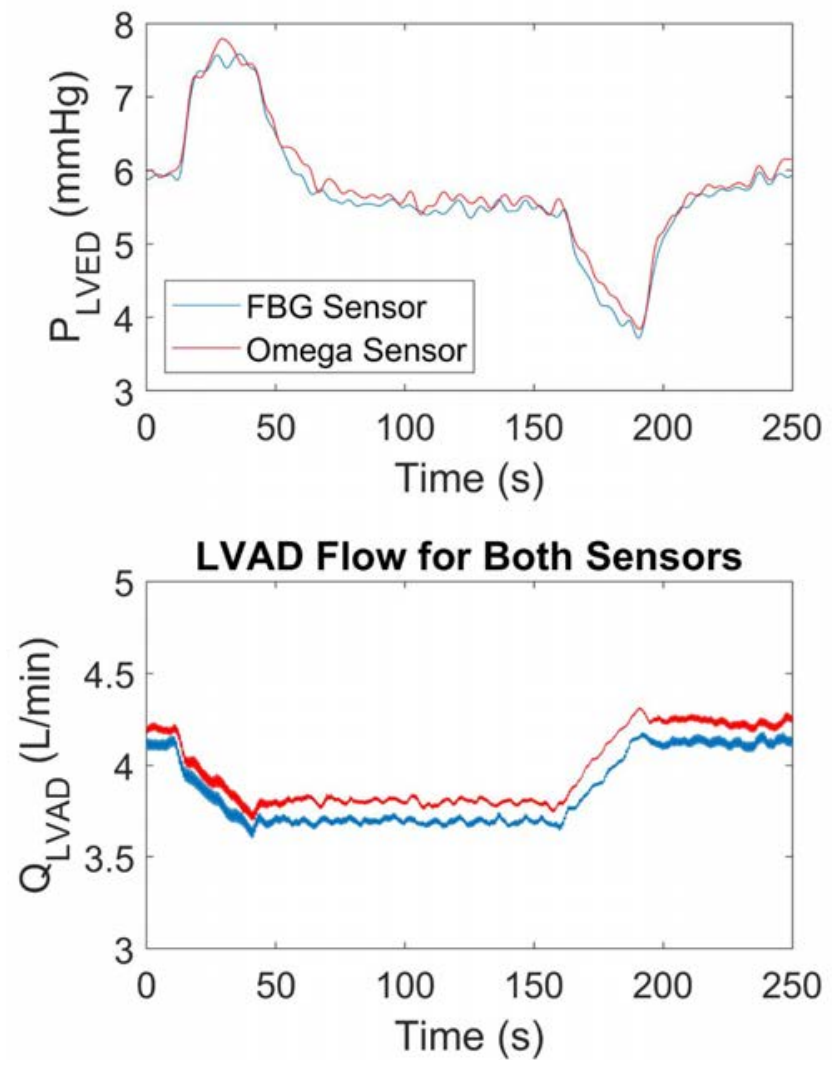

Fig. 8. End-diastolic pressure $\left(P_{\mathrm{LVED}}\right)$ (top) and mean LVAD flow $\left(\overline{Q_{\mathrm{LVAD}}}\right)$ (bottom) for Starling-like control with feedback from the FBG sensor (blue) and Omega sensor (red) during increases in SVR.

\section{Decrease in Preload}

The decrease in preload caused the controller to decrease VAD speed and flow for both of the feedback modalities. There were some discrepancies between the OP paths, with the Omega-SLC producing a larger OP path area than the FBG-SLC (1.68 and $1.23 \mathrm{~L} \cdot \mathrm{mmHg} / \mathrm{min}$, respectively). Both feedback modalities decreased $\overline{Q_{\mathrm{LVAD}}}$ to $3.7 \mathrm{~L} / \mathrm{min}$. 


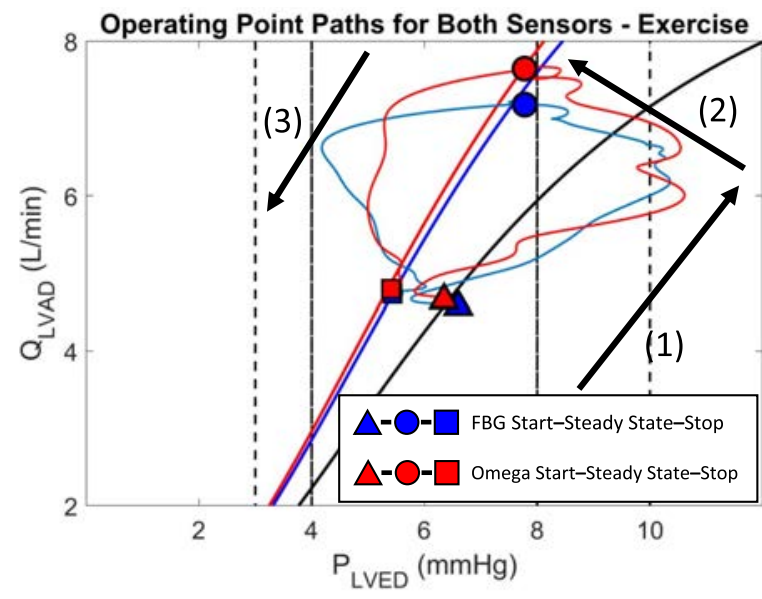

Fig. 9. SLC OP path using FBG transducer (FBG-blue) and Omega transducer (red) as a feedback variable during the onset of exercise. The arrow labeled (1) indicates the OP path following the onset of exercise, the arrow labeled (2) indicates the OP path following a control line adaption, and the arrow labeled (3) shows the OP path following return to resting condition. The markers indicate the start, steady state, and final positions of the OP. The dotted lines indicate the control box; the solid black line is the original Starling-like control line.

The steady-state $P_{\text {LVED }}$ was 5.6 and $5.5 \mathrm{mmHg}$ for FBG-SLC and Omega-SLC, respectively.

\section{E. Simulated Exercise}

During simulated exercise, there was a potentially clinically relevant difference between the controller OP path areas $(9.4 \mathrm{~L} \cdot \mathrm{mmHg} / \mathrm{min}$ for FBG-SLC and $10.30 \mathrm{~L} \cdot \mathrm{mmHg} / \mathrm{min}$ for Omega-SLC). Small variations in the $P_{\mathrm{LVED}}$ between the Omega and FBG pressure sensor $(10.3$ and $10.5 \mathrm{mmHg}$, respectively) resulted in slightly different control lines following emergency adaption (1.28 for the FBG-SLC and 1.32 for the Omega-SLC), which occurred for both feedback modalities (see Fig. 9). This resulted in a higher steady-state flow for the Omega-SCL $(7.7 \mathrm{~L} / \mathrm{min})$ compared with the FBG-SLC (7.2 L/min); this demonstrates how pressure error can be compounded in the control line calculation (see discussion). The steady-state $P_{\mathrm{LVED}}$ following SS adaption was $7.8 \mathrm{mmHg}$ for both feedback modalities. Despite the relatively large changes in flow, no transient difference in controller settling time was recorded.

\section{DISCUSSION}

The FBG sensor was used to provide feedback to an SLC, which was evaluated in a VAD supported MCL under changes in preload, afterload, and exercise. The control performance of Starling-like control using the FBG sensor for feedback was compared against the control performance of the SLC using an Omega sensor for feedback. For changes in preload and afterload, the average difference in steady-state $\overline{Q_{\mathrm{LVAD}}}$ between the two feedback methods was $-0.1 \mathrm{~L} / \mathrm{min}$, with a maximum steady-state difference of $-0.4 \mathrm{~L} / \mathrm{min}$ during exercise. The average difference in steady-state $P_{\text {LVED }}$ between the two feedback methods was $0.02 \mathrm{mmHg}$, with a maximum difference of $-0.2 \mathrm{mmHg}$ during increased preload. Given that the differences in $P_{\mathrm{LVED}}$ and $\overline{Q_{\mathrm{LVAD}}}$ are clinically insignificant, it has been shown that the FBG pressure sensor is effective as feedback for the SLC.

Despite the steady-state values being clinically similar, the OP areas and OP paths were different between the FBGSLC and Omega-SLC. The FBG sensor was less reactive (as demonstrated by smaller OP path areas) during all scenarios except increase in SVR; this is likely explained due to the higher compliance of the FBG pressure sensor, resulting in less variable transient pressure (as high compliance leads to a more damped pressure response) compared with the Omega pressure sensor that has very low compliance. Although there were differences in the transient response of the feedback methods, no difference in overall settling time of the system was recorded for any experiment, likely due to compensation through the PI controllers that control the VAD speed. Although there were sometimes large differences in terms of the absolute percentage, the variations in OP paths between the two feedback methods would not likely result in different clinical outcomes for the patient, with the possible exception of the exercise condition.

The adaptive nature of the SLC compounded the differences between the two feedback methods. For instance, during exercise, there was a $0.2-\mathrm{mmHg}$ difference between the transient $P_{\mathrm{LVED}}$ readings between FBG-SLC and OmegaSLC. Although, in absolute terms, this error was small, it contributed to the calculation of the new scaling factor following adaption, leading to a higher control line for the Omega-SLC (scaling factor $=1.32$ ) than its FBG counterpart (scaling factor $=1.28$ ). This, in turn, resulted in a steadystate flow difference of $0.4 \mathrm{~L} / \mathrm{min}$, which may be clinically significant. However, given that the OP of both of the control modalities was still within the SS zone, it can be argued that the patient was "optimally" managed according to the SLC configuration, and therefore, the small discrepancies would not be harmful. In addition, it is interesting to note that following the alleviation of the exercise condition, there was no steady state difference between $P_{\mathrm{LVED}}$ or $\overline{Q_{\mathrm{LVAD}}}$ of the two feedback modalities; this was due to the convergence of the control lines at low preloads.

Previously, only two fully described experimental pressure sensors have been incorporated into the VAD inlet and used for physiological control purposes [28], [29]. Both studies used a novel MEMs diaphragm-based pressure sensor that could be incorporated directly into a VAD inlet. The first study used a multiobjective controller that used VAD inlet and outlet pressure, as well as heart rate to maintain a set level of ventricular filling pressure and outlet pressure [28]. Although the study stated that a catheter-based pressure sensor was used as a reference sensor, the study did not report the differences between the developed and reference sensors during control and did not use the reference sensor for controller feedback. The controller in the second study used measured ventricular pulsatility as a measure of preload and aimed to keep the pulsatility at a constant set point [29]. That study also used a catheter-based pressure sensor as a reference sensor and reported a differential between the measured left ventricular pressure and pump inlet pressure due to friction losses in the cannula (as the developed and reference sensors were not 
placed at the same distance along the cannula). The authors of the aforementioned study did not directly compare the controller performance between the developed and reference sensors.

Optical sensing of pressure may also be achieved using the Fabry-Perot interferometry using highly reflective surfaces at the tip of the optical fiber. Interferometry may seem like a more logical choice for measuring VAD control, given that it measures pressure directly at the fiber without the need for a transduction medium. A previous study investigated the use of Fabry-Perot interferometry for measuring VAD inlet pressure [16]. In that study, the Fabry-Perot pressure sensor demonstrated a sensitivity of $0.163 \mu \mathrm{m} / \mathrm{mmHg}$ and a resolution of $1 \mathrm{mmHg}$. By comparison, the FBG pressure sensor used in this study is much more sensitive at $-0.0046 \mathrm{~nm} / \mathrm{mmHg}$ and has a better resolution of $0.019 \mathrm{mmHg}$. The much higher resolution and sensitivity of the FBG technology mean that it has the potential to be embedded directly into more rigid materials, including the possibility of embedding it directly into the titanium of the VAD inlet, thereby reducing or eliminating an interface for thrombus to grow.

In this study, $P_{\mathrm{LVED}}$ used as an indicator of preload was actually the pump inlet pressure. In reality, the pump inlet pressure is lower than the ventricular pressure due to the pressure gradient along the VAD inlet cannula due to friction losses. This will lead to pressure readings, which may be lower than the actual ventricular pressure posing a potential increased risk of pulmonary congestion, though still less so than constant speed VAD control. The effects of the pressure gradient will increase further away that the FBG sensor is placed from the ventricle and the gradient may also be increased with a shorter cannula. Placing the sensing element as close to the ventricle as possible will result in the closest measurement to the actual ventricular pressure, or it may also be possible to mathematically compensate for the gradient in the cannula to predict the ventricular pressure using the Hagen-Poiseuille equation. The Hagen-Poisulle equation will, however, be limited, as it suffers from the assumptions that there is no inflow cannula thrombus, and that blood is a Newtonian fluid-which it is not. Compensation could also be achieved dynamically by placing a sensing element at either end of the cannula with a fixed distance between them and calculating the pressure differential between the two, as suggested for previous VAD inlet pressure sensors [28].

The main limitation of this experiment is that it was conducted in an MCL. While MCLs allow physiologically representative, cost-effective, repeatable results, they cannot model all of the biological processes. The MCL employs a passively filled, rigid mock ventricle. However, the native ventricle has more variable compliance than the rigid MCL ventricle. The compliances of the ventricle and FBG sensor can be modeled simply by two capacitors in parallel, which would make the compliance of the ventricle and pressure sensor additive. Further experiments will need to be conducted with the sensor in vivo to determine if the higher compliance of the native ventricle will produce unpredictable pressure readings by the FBG, which would lead to unreliable controller behavior.
The changes in preload and afterload in these experiments were severe, and the onset was more rapid than would typically occur in a VAD supported patient. The resulting hemodynamic values were, therefore, also quite extreme (arterial pressure ranging 48-170.9 $\mathrm{mmHg}$ ) and not representative of normal physiological parameters. Given the FBG-SLC's stability even under these circumstances, it can be assumed that the FBGSLC will perform adequately under milder, physiological condition changes.

The sensor and SLC both require further work in preparation for preclinical trials. Further work on the sensor will involve the improvement of sensor biocompatibility with enhanced integration of the fiber optic interrogator into the VAD driveline, which connects the VAD to the pump power source and controller. Improvement of biocompatibility will involve research into flexible biocompatible and biostable materials and advanced manufacturing techniques. The integration of the fiber optic interrogator will require research into the photoelectronic device miniaturization and should also investigate native integration between the FBG sensor and SLC. It would be ideal to produce a VAD controller that has the SLC algorithm integrated, which is able to energize the VAD, and also has some integrated hardware for reading the FBG pressure sensor as well as a flow sensor. This control unit could be used for both monitoring and control purposes. The creation of a single control unit would allow for all of the peripherals (VAD, pressure sensor, and flow sensor) to be integrated into the driveline, resulting in one exit site and no internal batteries.

\section{Conclusion}

This study aimed to demonstrate the feasibility of using a novel FBG sensor as feedback for an SLC. The FBG provided continuous, real-time feedback to the SLC, and the clinically insignificant differences between the steady-state outputs of the two feedback modalities were attributed to acceptable signal noise and a difference in the compliance between the two sensors. This study demonstrates that the FBG sensor is acceptable for physiological control applications. Further work will be required on both the FBG sensor and SLC to demonstrate clinical readiness. A successful development of an implantable continuous pressure sensor, such as the one presented in this study, will solve the major technical limitation, which has hindered physiological control of VADs for the last 20 years.

\section{REFERENCES}

[1] J. K. Kirklin et al., "Eighth annual INTERMACS report: Special focus on framing the impact of adverse events," J. Heart Lung Transplantation, vol. 36, no. 10, pp. 1080-1086, Oct. 2017.

[2] D. C. Chambers et al., "The registry of the international society for heart and lung transplantation: Thirty-fourth adult lung and heart-lung transplantation report-2017; focus theme: Allograft ischemic time," J. Heart Lung Transplantation, vol. 36, no. 10, pp. 1047-1059, Oct. 2017.

[3] J. G. Rogers et al., "Continuous flow left ventricular assist device improves functional capacity and quality of life of advanced heart failure patients," J. Amer. College Cardiol., vol. 55, no. 17, pp. 1826-1834, Apr. 2010.

[4] M. Modica et al., "Quality of life and emotional distress early after left ventricular assist device implant: A mixed-method study," Artif. Organs, vol. 39, no. 3, pp. 220-227, Mar. 2015. 
[5] J. A. Cowger et al., "Quality of life and functional capacity outcomes in the MOMENTUM 3 trial at 6 months: A call for new metrics for left ventricular assist device patients," J. Heart Lung Transplantation, vol. 37, no. 1, pp. 15-24, Jan. 2018.

[6] R. F. Salamonsen, D. G. Mason, and P. J. Ayre, "Response of rotary blood pumps to changes in preload and afterload at a fixed speed setting are unphysiological when compared with the natural heart," Artif. Organs, vol. 35, no. 3, pp. E47-E53, Mar. 2011.

[7] M. Granegger, T. Schlöglhofer, H. Ober, D. Zimpfer, H. Schima, and F. Moscato, "Daily life activity in patients with left ventricular assist devices," Int. J. Artif. Organs, vol. 39, no. 1, pp. 22-27, Jan. 2016.

[8] A. F. Stephens, M. C. Stevens, S. D. Gregory, M. Kleinheyer, and R. F. Salamonsen, "in vitro evaluation of an immediate response starling-like controller for dual rotary blood pumps," Artif. Organs, vol. 41, no. 10, pp. 911-922, Oct. 2017.

[9] A. Petrou, J. Lee, S. Dual, G. Ochsner, M. Meboldt, and M. Schmid Daners, "Standardized comparison of selected physiological controllers for rotary blood pumps: In vitro study," Artif. Organs, vol. 42, no. 3 , pp. E29-E42, Mar. 2018.

[10] J. P. Pauls et al., "in vitro comparison of active and passive physiological control systems for biventricular assist devices," Ann. Biomed. Eng., vol. 44, no. 5, pp. 1370-1380, May 2016.

[11] G. Sayer, V. Jeevanandam, T. Ota, and N. Uriel, "Invasive hemodynamic echocardiographic ramp test in the HeartAssist5 LVAD: Insights into device performance," ASAIO J., vol. 63, no. 2, pp. 10-12, 2017.

[12] D. Banerjee, D. Dutt, S. Duclos, K. Sallam, M. Wheeler, and R. Ha, "Simultaneous ramp right heart catheterization and echocardiography in a ReliantHeart left ventricular assist device," World J. Cardiol., vol. 9, no. 1, pp. 55-59, 2017.

[13] E. Bullister, S. Reich, P. d'Entremont, N. Silverman, and J. Sluetz, "A blood pressure sensor for long-term implantation," Artif. Organs, vol. 25 , no. 5, pp. 376-379, May 2001.

[14] S. Staufert and C. Hierold, "Novel sensor integration approach for blood pressure sensing in ventricular assist devices," in Proc. 30th Eurosensors Conf., vol. 168, 2016, pp. 71-75.

[15] L. Brancato, G. Keulemans, T. Verbelen, B. Meyns, and R. Puers, "An implantable intravascular pressure sensor for a ventricular assist device," Micromachines, vol. 7, no. 8, pp. 1-17, 2016.

[16] M. D. Zhou, C. Yang, Z. Liu, J. P. Cysyk, and S. Y. Zheng, "An implantable Fabry-Pérot pressure sensor fabricated on left ventricular assist device for heart failure," Biomed. Microdevices, vol. 14, no. 1, pp. 235-245, 2012.

[17] S. Poeggel, D. Tosi, D. Duraibabu, G. Leen, D. McGrath, and E. Lewis, "Optical fibre pressure sensors in medical applications," Sensors, vol. 15, no. 7, pp. 17115-17148, 2015.

[18] A. F. Stephens, A. Busch, R. F. Salamonsen, S. D. Gregory, and G. D. Tansley, "A novel fibre Bragg grating pressure sensor for rotary ventricular assist devices," Sens. Actuators A, Phys., vol. 295, pp. 474-482, Aug. 2019.

[19] K. O. Hill, Y. Fujii, D. C. Johnson, and B. S. Kawasaki, "Photosensitivity in optical fiber waveguides: Application to reflection filter fabrication," Appl. Phys. Lett., vol. 32, no. 10, p. 647, 1978.

[20] A. F. Stephens, A. Busch, S. D. Gregory, R. F. Salamonsen, and G. Tansley, "Temperature compensated fibre Bragg grating pressure sensor for ventricular assist devices," in Proc. 40th Annu. Int. Conf. IEEE Eng. Med. Biol. Soc., Jul. 2018, pp. 1-4.

[21] A. Stephens, S. Gregory, G. Tansley, A. Busch, and R. Salamonsen, "In vitro evaluation of an adaptive Starling-like controller for dual rotary ventricular assist devices," Artif. Organs, vol. 43, no. 11, pp. E294-E307, Nov. 2019.

[22] E. H. Starling and M. B. Visscher, "The regulation of the energy output of the heart," J. Physiol., vol. 62, no. 3, pp. 243-261, 1926.

[23] A. C. Guyton, "Determination of cardiac output by equating venous return curves with cardiac response curves," Physiol. Rev., vol. 35, no. 1 , pp. 123-129, Jan. 1955

[24] S. J. Sarnoff and E. Berglund, "Ventricular function: I. Starling's law of the heart studied by means of simultaneous right and left ventricular function curves in the dog," Circulation, vol. 9, no. 5, pp. 706-718, May 1954.

[25] A. F. Stephens, S. D. Gregory, and R. F. Salamonsen, "The importance of venous return in Starling-like control of rotary ventricular assist devices," Artif. Organs, vol. 43, no. 3, pp. E16-E27, Mar. 2019.

[26] S. D. Gregory et al., "An advanced mock circulation loop for in vitro cardiovascular device evaluation,” Artif. Organs, Jan. 2020.
[27] J. P. Pauls, L. A. Roberts, A. Stephens, J. F. Fraser, G. Tansley, and S. D. Gregory, "Improving in vitro evaluation capabilities of cardiac assist devices through a validated exercise simulation," in Proc. 41st Аnnu. Int. Conf. IEEE Eng. Med. Biol. Soc. (EMBC), Jul. 2019, pp. 4901-4904.

[28] E. Bullister, S. Reich, and J. Sluetz, "Physiologic control algorithms for rotary blood pumps using pressure sensor input," Artif. Organs, vol. 26, no. 11, pp. 931-938, Nov. 2002.

[29] J. Cysyk, C.-S. Jhun, R. Newswanger, W. Weiss, and G. Rosenberg, "Rotary blood pump control using integrated inlet pressure sensor," in Proc. Annu. Int. Conf. IEEE Eng. Med. Biol. Soc., Aug. 2011, pp. 373-376.

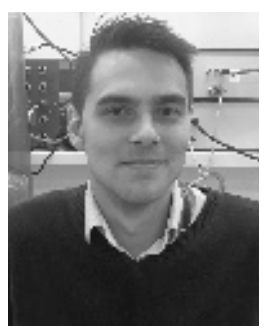

Andrew F. Stephens received the Ph.D. degree in control of ventricular assist devices from Griffith University in 2019.

$\mathrm{He}$ is currently a Research Fellow with Monash University, Clayton, VIC, Australia, and the Deputy Director of the Cardio-Respiratory Engineering and Technology Laboratory (CREATELab), Melbourne, VIC. He is also an electronic and computer engineer. His research focus is on physiological control of cardiorespiratory devices, patient instrumentation, and monitoring.

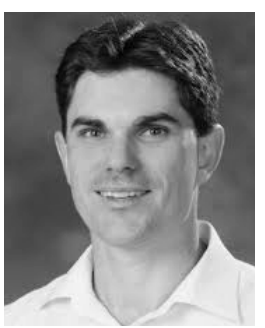

Andrew Busch (Member, IEEE) received the Ph.D. degree in machine vision and computer learning from the Queensland University of Technology in 2004.

$\mathrm{He}$ is currently a Senior Lecturer with the School of Engineering and Built Environment, Griffith University, Brisbane, QLD, Australia. He is also an electronic and computer engineer. His research focus is on automation through computer vision and machine learning.

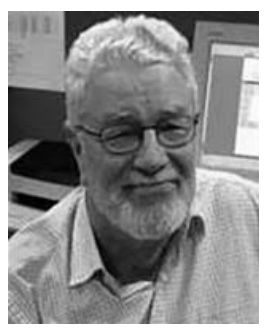

Robert F. Salamonsen is a Senior Consultant with the Intensive Care Unit, Alfred Hospital, Melbourne, VIC, Australia. He holds an emeritus professorship with Monash University, Melbourne. His research focus is on automatic control of ventricular assist devices.

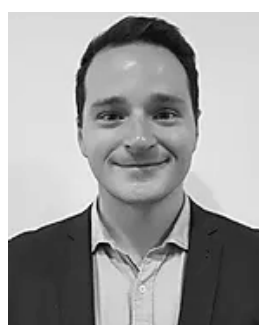

Shaun D. Gregory received the Ph.D. degree in novel fixation devices for ventricular assist device cannulae from the Queensland University of Technology in 2009.

He is currently a National Heart Foundation Future Leader Fellow and a Senior Lecturer with the Department of Mechanical and Aerospace Engineering, Monash University, Clayton, VIC, Australia. $\mathrm{He}$ is also the Laboratory Director of the CardioRespiratory Engineering and Technology Laboratory (CREATELab), Melbourne, VIC. He is a mechanical and biomedical engineer. His research focus is on mechanical cardiorespiratory technology and device evaluation.

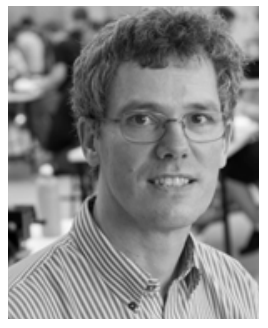

Geoffrey D. Tansley received the Ph.D. degree in mechanical engineering from Nottingham Trent University, Nottingham, U.K., in 1988.

$\mathrm{He}$ is currently a Professor with the School of Engineering and Built Environment, Griffith University, Gold Coast, QLD, Australia. He is also a mechanical engineer. His research focus is on mechanical circulatory support and the development of novel medical and industrial devices. 